\title{
Predatory monetisation? A categorisation of unfair, misleading, and aggressive monetisation techniques in digital games from the perspective of players
}

Elena Petrovskaya, University of York

David Zendle, University of York

\begin{abstract}
Technological shifts within the video game industry have enabled many games to evolve into platforms for repeated expenditure, rather than a one-time purchase product. Monetising a game as a service is challenging, and there is concern that some monetisation strategies may constitute unfair or exploitative practices. We asked 1104 players of video games to describe a time when they had been exposed to transactions which were perceived to be misleading, aggressive or unfair. We found 35 separate techniques over 8 domains: game dynamics designed to drive spending, product not meeting expectations, monetisation of basic quality of life, predatory advertising, in-game currency, pay to win, general presence of microtransactions and other. Notably, several of these reported practices seem to not align with existing UK consumer protection regulations. We discuss this potential misalignment, as well as the implications of identifying what players believe to be problematic monetisation techniques.
\end{abstract}

Keywords: predatory monetisation, consumer protection, microtransactions, video games, in-game purchasing.

\section{Introduction}

The norm for revenue streams in the games industry used to be of a one-time purchase model, in which upfront payment was the only time a transaction was needed to guarantee a full gameplay experience (Lizardi, 2012). As underpinning technology emerged to facilitate continuous payment, monetisation approaches have evolved that treat games as potential sources for consistent expenditure, rather than a product in and of themselves. Since then, business models in the games industry have moved through several models, including pay-per-play, shareware, and subscription (Alha, 2020; Paul, 2020). Currently, one of the most popular ways of monetising games is the so-called 'freemium' model, in which core game content is available for free, and revenue generation takes place entirely through the sale of additional features or advantages during play. Such sales typically take the form of microtransactions - unrestricted in-game payments which players can make at any point (Schwiddessen \& Karius, 2018). Microtransactions are generally divided into decorative, which affect purely in-game appearance, or functional, which affect gameplay (Oh \& Ryu, 2007).

This diversification of monetisation beyond the sale of games as products has proven lucrative for the video game industry. Indeed, in one quartile of 2019 alone, just the company EA made over \$1 billion from microtransactions (Narayan, 2020). With that comes the concern that novel approaches to video game monetisation may be implemented in ways that are exploitative, unethical, or not in the best interests of gamers (Alha, Koskinen, Paavilainen, Hamari, \& Kinnunen, 2014). King and Delfabbro (2018) discuss the notion of 'predatory monetisation,' referring to schemes which involve in-game purchase systems that disguise or withhold the true long-term cost of the activity until players are psychologically or financially committed. Such manipulation is described as using elements of intrusive solicitations, limited disclosure, and manipulation of reward outcomes. 
The example of predatory monetisation which has received the most regulatory attention is that of loot boxes - items in video games that may be bought for real-world money, but which provide players with a randomised reward of uncertain value (Zendle, Ballou, \& Meyer, 2019). Loot boxes have attracted attention primarily because of their randomisation element, which has been linked to problem gambling (Zendle \& Cairns, 2018). This has raised concerns regarding player wellbeing, particularly in vulnerable groups, such as children and adolescents (Zendle, Meyer, \& Over, 2019).

Besides links to potentially negative consequences for player wellbeing, there are other ways in which monetisation techniques may have negative impact. Consumer protection refers to the idea that buyers should be safeguarded against unfair or exploitative marketplace practices (GOV.UK, 2020). In the United Kingdom, for example, the Consumer Protection from Unfair Trading Regulations 2008 (hence 'Unfair Trading Regulations') are designed to protect consumers by prohibiting unfair, misleading, and aggressive business practices (The Consumer Protection from Unfair Trading Regulations 2008, 2008). Contravention of the Regulations may mean consumers engaged in a transaction because of processes outside of their own free will, and may also mean their experience of a product (in this context, of a game), is distorted or impacted negatively. The way that these regulations intersect with contemporary monetisation in video games is, however, currently unclear.

It seems likely that players of games themselves may provide a rich resource in understanding the consequences of money-making in video games. Players regularly interact with in-game transactions, and therefore are likely to have in-depth understanding and nuanced perspectives on when these transactions might be considered misleading or unfair. Indeed, members of the player community themselves have attempted to build up informal classifications of monetisation techniques (Shokrizade, 2013), indicating that such knowledge is important and interesting to the gamer as well as the research community.

Furthermore, players have been used as a data source in previous research in the domain. Lin \& Sun (2011) carried out an analysis of forum data from two of the most popular game bulletin boards in Taiwan, as well as game magazines and player interviews to understand how the free-to-play model may affect the player experience along dimensions of fairness, immersion, and others. Their findings showed that this business model contributed to a shift of player self-perception in their relationship with games, as well as their perceptions of games and the communities which they attract. Similarly, Alha's et al.'s (2018) interview study asked 11 players about their perceptions and experiences of free-to-play games, and found that although as a whole, the model was not perceived negatively, certain aspects of in-game payments were seen as more problematic, for example, some players were conflicted about payment for advancement.

Besides investigating player perspectives on in-game purchases, research has been conducted on defining the different forms that such purchases may take. For example, Windleharth and Lee (2020) recruited a sample to play 65 mobile games, and generated two taxonomies from this play: the types of transactions between game players and companies that 'transfer or create value for the gamer owner', and a 'taxonomy of methods companies use to drive engagement and retention with mobile games.' Other work has included an examination of video game patents (King et al., 2019), and the examination of games from a design perspective to identify 'dark patterns' in the way monetisation has been implemented (Dahlan, 2020; Zagal, Björk, \& Lewis, 2013). Hamari et al. (2017) incorporated the players into their taxonomisation, constructing six in-game purchase motivation dimensions based on a survey of 519 people.

Our work continues the methodology of Hamari et al. (2017) and builds on the research directions of Alha (2018) and Lin and Sun (2010) by developing a taxonomy of player-perceived problematic in-game purchases through a qualitative survey of players. This also aligns with recent recommendations for doing policy-relevant research in psychological sciences to draw on stakeholders for identification of problems (IJzerman et al., 2020). By drawing on player perspectives to characterise 
potentially problematic in-game microtransactions, we have access to information which would be difficult to glean externally, and are able to understand the consumer experience for games as a product.

\section{Methodology}

We use the UK's Unfair Trading Regulations as the basis for our definition: simply using the word 'problematic' when gathering player opinions would likely have led to a discrepancy in people's understanding.

Operationalising 'problematic' in-game transactions as 'unfair', 'misleading' or 'aggressive' according to an established legal definition provided a clear reference point. These three words were used when asking players in the survey about their experiences with in-game transactions to elicit responses. According to the Regulations, a commercial practice is unfair if it "contravenes the requirements of professional diligence" and "materially distorts or is likely to materially distort the economic behaviour of the average consumer with regard to the product" - meaning it may affect consumer decisions regarding whether to purchase the product. In a similar vein, misleading practices involve using untruthful information for the presentation of a product, which is likely to impact consumer perception of the product and subsequently their decision. Misleading actions include misleading advertising, artificial scarcity, false information, and misleading omissions, in the case of which certain information about the product is withheld. An aggressive tactic is one which "significantly impairs or is likely significantly to impair the average consumer's freedom of choice or conduct in relation to the product concerned through the use of harassment, coercion or undue influence" and as such again "causes or is likely to cause him to take a transactional decision he would not have taken otherwise" (The Consumer Protection from Unfair Trading Regulations 2008, 2008). In short, the Regulations outline practices according to which it is believed a consumer decision about whether to purchase a product is inappropriately affected by the provider of the product. A trader would be committing offence by engaging in any of the above practices.

The idea that consumer protection is a useful lens for studying microtransactions has been suggested by past research (King et al., 2019), and by assessing video game monetisation via reference to a previously-agreed-upon standard for consumer protection rather than seeking to define novel harms, one may suggest that this work provides greater clarity and use value from a regulatory perspective.

\section{Design}

Data were collected through an online survey. Questions asked players about transactions they had encountered which they felt had misled them, and how that experience had made them feel, and then likewise about transactions which they felt had been unfair or aggressive towards them. As described above, these words were taken directly from the Unfair Trading Regulations. The questions were open, for example 'Think of any features you may have encountered in games, the end goal of which was a transaction of real money, that you feel misled you - gave you the wrong idea or impression - in order to promote the transaction'. The aim of this question was to gather information about as many techniques as possible. Some additional questions around game monetisation were also included but not used in the current analysis. Players were also asked how long they had been playing games, how long they had been playing games, an example of a game they had been playing a lot recently and an example of a favourite game. Other background characteristics, such as age or gender, were not collected - we wanted to prioritise truthful and open discussion of monetisation experiences, and felt that asking for less anonymity may have impeded this. The full survey is available at tinyurl.com/predatorymon.

Note: as mentioned earlier in this text, loot boxes are already well-established in the literature as a potentially problematic form of video game transaction. In order to prevent respondents from focusing on this aspect of game monetisation, an 
initial question in the survey asked players to name games in which they had seen specifically loot boxes. Prior to open-ended questions about problematic monetisation participants were then told "The next section will ask you about any in-game transactions you may have encountered besides lootboxes".

\section{Participants}

Participants were recruited using the discussion website Reddit and the social networking website Twitter. To ensure we had a sample of players representing a wide breadth of games, we used a list of 100 most played Steam games, and 100 most played mobile games, and found the subReddits (special interest online bulletin boards) for all of these games. The moderators of all the individual subReddits were then contacted to ask for permission to post the survey. In the end, we were given permission to post on 50 game-specific subReddits, of which 19 were mobile and the rest were PC. In addition to this, we were able to post on more general game-related subReddits: $r$ /Steam, $r$ /Twitch, $r$ /PCMasterRace, $r /$ TrueGaming, $r /$ MobileGaming, $r$ /SampleSize. One must note that we did not restrict our game samples to free-to-play games only, as many paid games also incorporate microtransactions, and we were interested in problematic in-game purchases across all games. We also publicised the survey on Twitter. We believe this sampling frame allowed for a diverse sample of players of different games. The full list of subReddits is available at tinyurl.com/predatorymon.

In total, 1471 respondents completed the survey. After data cleaning and processing (as none of the questions were compulsory due to ethical considerations), 1104 were included in the analysis. It is of interest that respondents were highly engaged with this research: we received numerous positive comments and responses to the Reddit posts, as well as some direct emails sharing experiences and asking for the results when they were available. Several participants even offered to publicise the survey around their own networks.

\section{Analysis}

Content analysis (Mayring, 2004) was used as an analysis method to identify patterns and classify the techniques presented by respondents into categories. Each mentioned technique was coded, regardless of how many were mentioned by each respondent, or in each utterance. Two coders, both of whom can be justified as being experts in the monetisation domain, worked together to develop a categorisation. This was done by initially separate coding, with regular discussions. Once both coders had developed separate coding schemes, they met to merge the codes and resolve any discrepancies. This resulted in the development of a final shared categorisation system by the first coder, which the second coder signalled their agreement with.

An independent rater who had not been related in the generation of the codebook, but was familiar with the subject matter, coded a subset of the data (100 utterances) against the codebook. The same subset was coded by one of the original coders, and a Kappa score, a standardised measure of inter-rater reliability, was calculated. A Kappa statistic of greater than or equal to 0.81 is classed as being 'almost perfect agreement' (McHugh, 2012). For this reason, and to keep consistency with our previous work, we set a minimum threshold of agreement of 0.81 .

In the first pass, the Kappa score was 0.62 , which did not meet our proposed benchmark of 0.81 . We were able to identify some trends in disagreement, which were resolved through an iteration of the codebook. The same independent rater was asked to code a fresh subset of 100 utterances, and the process was again repeated by one of the original coders. In the second iteration, the Kappa score was 0.92 . We believed this to be a high enough score to be confident in the reliability of our results. However, before finalising the categories, the two authors discussed the codes which had contributed to the remaining rater disagreements, and made final edits to their phrasing. All of the data was then re-coded using the final coding scheme in order to have accurate numbers of occurrences and examples. Alongside doing this, a third sample of 100 
utterances was coded by the same independent rater, and the final inter-rater reliability was 0.84 . The final codebook is available at tinyurl.com/predatorymon.

\section{Results}

In total, 35 monetisation issues were reported by players as being either misleading, unfair, or aggressive. We have grouped these issues into eight overall domains (Figure 1):

1. Game dynamics designed to drive spending

2. Product not meeting expectations.

3. Monetisation of basic quality of life.

4. Predatory advertising.

5. In-game currency.

6. Pay to win.

7. General presence of microtransactions.

8. Other.
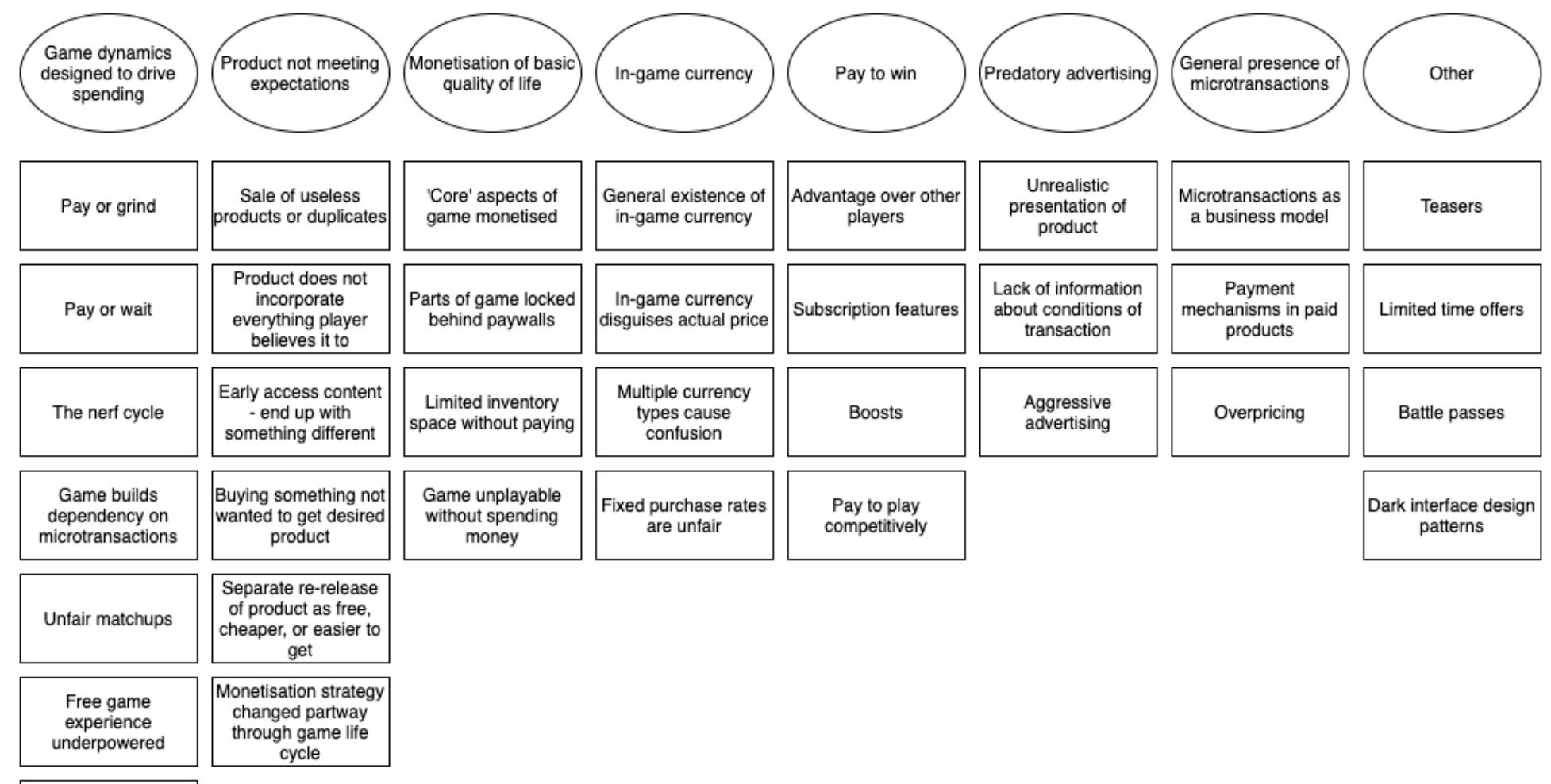

Payment needed to avoid negative consequences

Figure 1. A visual illustration of the monetisation issues reported by players as misleading, unfair, or aggressive.

\section{Game dynamics designed to drive spending}

These are situations where players feel game dynamics - the ways in which the game patterns and players evolve over time have been designed especially to encourage spending, rather than primarily for the improvement of a player's in-game experience. 


\section{Pay or grind}

Gamers perceive themselves as being given the binary choice of either investing an unpleasantly large amount of time and effort into completing a portion of the game, or completing a transaction to avoid having to invest the same extent of time and effort. Players often reported feeling like a game has specifically been designed in this way to push them into the seemingly easier option of spending to circumvent grind timers. This was a prominent mechanism $(N=57)$.

"In Darkorbit you have to either spend insane amounts of money, spend insane amounts of time grinding or buy a third party software that grinds for you. "(P239)

Furthermore, players often appeared to resent the manipulation of the game in this way.

"Upgrades to skip grinding defeats the purpose of video games that give feeling [sic] of achievement." (P276)

\section{Pay or wait}

In contrast to pay or grind, which involves payment to avoid the expenditure of time and effort, this refers to a situation in which players are given the choice of waiting some time before being able to progress in the game, or paying some money to skip this wait.

"Everything you did had obsorbetently [sic] long wait times with the option to speed them up by spending premium currency." (P927)

\section{The nerf cycle}

Another commonly expressed idea $(N=35)$, referring to a situation in which players perceive an item of specific strength or usefulness being sold at specific price, only for that item to be reduced in strength or general value at a point after the transaction. Frequently, this process of 'nerfing' is described as a cyclical, deliberate system: a new item is released, then depowered ('nerfed') in preparation for another new release.

"On the other hand War Thunder by Gaijin offers a wide range of premium vehicles, which break the game balance upon release, to make them more attractive, and then are "nerfed" (weakened or rebalanced) which is very questionable. It seems that Gaijin wants WT to earn money by selling more and more tanks which are slowly nerfed, while new overpowered tanks are released again soon after." (P717)

\section{Game builds dependency on microtransactions}

In some cases, players feel that as they progress through a game, they feel pressured to spend progressively more and more to have a good gameplay experience. This kind of increasing dependency is perceived as sometimes being implemented through providing a resource (e.g. game speed or currency) more freely at the beginning of a game, and then reducing the availability of that resource during play.

"When mobile games offer some premium currency in abundance at the beginning of your exposure to the game, and then decline the amount available as time moves forward. This practice misrepresents the abundance of this resource, driving the player to make a transaction to gain a sense of normalcy and power which they had at the start of the experience." (P140) 
"I generally know what I'm getting into when spending real money, but dramatically escalating in-game costs are what annoy me. Ex. You buy magic bucks to get an upgrade only to find out the next upgrade would require significantly more magic bucks to accomplish." (P584)

\section{Unfair matchups}

Some players who are playing using only free in-game items believe they are consistently matched against opponents who have an advantage from buying items, which makes the game experience unpleasant.

"Back when I played mobile games including player vs player elements (such as C.a.t.s., and Clash royale) I oftentimes found myself matched up with players that had opted to buy into the microtransaction store and found myself at an unfair disadvantage." (P780)

\section{Game experience is underpowered without in-game spending}

A related situation occurred when players perceived that a game's free game experience is underpowered so that players will have a worse experience without spending money, and occasionally feel driven into purchases,

"Monster hunter world. Seemed like an enjoyable game and it was until $i$ found out that the starter weaponry the game provides is extremely underpowered to push you into finishing the main game and buying the dlc asap." (P44)

\section{Payment is needed to avoid negative consequences}

This refers to scenarios where a game forces a player to spend not to gain any additional in-game content, but so they do not lose something they already have, such as content, progress, or rewards.

"In Fire Emblem Heroes there is a game mode in which you are temporarily given four units that as you play levels will access to the best skills in the game without their base cost, usually the sacrifice of the original unit that has the skill. The only way to keep those characters is by spending $30 \$$ on an item called a "forma soul." (P284)

\section{Product does not meet expectations}

Players used the domain frequently (but not exclusively) to refer to the outcomes of in-game microtransactions, describing a variety of situations in which a purchased product did not serve their purpose in the way the player might have expected before engaging with a transaction.

\section{Sale of useless products or duplicates}

This refers to situations in which players purchase an in-game product, but that product does not work in the intended way either because of some of the characteristics of the player's existing items, or because of an in-game duplication mechanic.

"On Love Nikki there was a rather low cost promotion which was a comeback, the problem was whoever had already bought it would not be warned, making a second unnecessary purchase" (P62)

"Buying equipment not yet usable by my character (ad said you could use the same day) - Warframe" (P740) 
Product does not incorporate everything the player believes

Some players felt that the products that they bought were not strictly useless or duplicates, but nonetheless did not match the expectations that were created for them, or had to be bought blindly due to a lack of information.

"It was sold as you would get all post launch content but they recently released a truck that isn't a part of the season pass and they implied they're gonna do it again." (P897)

\section{Early access content - end up with something different to what was paid for}

A related idea is situations in which players may purchase specific early access content, but experience disappointment due to ending up with something different that they paid for, or just never receiving the completed version of the game.

"A limited edition version of the game at purchase that got you early access to play and more stuff to be named later but never was." (P620)

\section{Buying something not wanted to get a desired product}

Players also thought that instances where they had to buy something not wanted to get a desired product were unfair. This refers to situations where desirable purchases (e.g. discounts, upgrades, etc.) which are only available as a consequence of buying something else.

"RPG's or gacha's [sic] where you have to get multiple of the same unit to level up said unit so you get 4 or 5 so you can level up your 1 main dude but "o no turns out every character needs to be at the same level as your top man for them to fuse and level up" so now your 5 or so hour journey to get a good character just turned into a 35 or 40 hour trip for 1 character." (P757)

\section{Separate re-release of product as free, cheaper, or easier to get}

Players identified instances in which a product was locked behind an initial transaction, often in some kind of bundle or subscription, but then re-released separately (normally cheaper) outside of the original purchase setup, or is made free and publicly available.

"In general: buying the DLC and then developers giving it away. Mafia 3 recently put out a free update which gave out the entire DLC catalogue for free. Why buy games the year they come out, if they'll be fixed and in-full 12 months down the line, for cheaper?" (P43)

\section{Monetisation strategy changed partway through the game lifecycle}

This referred specifically to situations where microtransactions were introduced into a game which did not have them before, or introduced in additional amounts.

"Crash Team Racing Nitro-Fueled - the devs behind this game made it clear that there would be no micro transactions in the game before release. About a month or so after I purchased the game and it got good reviews, the game got micro transactions in the form of an in game store for skins. People like me who try to avoid games with micro transactions ended up purchasing the game because the developers behind the game lied about their intentions, waited for the reviews to come out so the reviews won't mention the microtransactions, and then put in the micro transactions." (P757) 


\section{Monetisation of basic quality of life}

This domain refers to situations in which players discuss aspects of games which are central to what they imagined their experience of playing that game to be, but which they cannot access without a transaction.

\section{'Core' aspects of game monetised}

This category refers to situations where parts of a game which players feel are integral to that game are inaccessible without spending. Sometimes players even suggest that a free game has been created in a purposefully incomplete form so that players are forced to spend money on making it more complete.

"They then added quality of life improvements behind paywalls like bottomless inventory. summonable storage and vendors without wasting time managing limited inventory, or traveling to sell, restock on items. I purchased a lot of these admittedly and regret doing so now." (237)

\section{Parts of game locked behind paywall}

Relatedly, one of the most mentioned techniques in the sample $(N=58)$ was that of parts of game content locked behind paywalls, in which parts of the game which players wish they had access to are locked behind payments. This covers both functional and decorative content.

"Pokemon sword and shield DLC felt very miss placed [sic], as a fan for over 16 years it felt dishonest to lock specific Pokemon behind a paywall...It felt misleading to try to exploit fans like this the slogan is gotta catch em all not pay to catch most of them..." (P86)

"Forza Horizon 4: In that game if you don't have all the dlc expansions, you will get missions on your map that you can travel to, but will not be able to participate because you're missing that content. This is extremely misleading and frustrating when you travel the whole size of the map only to realise that you cannot do the race."(P903)

\section{Limited inventory space without paying}

Some games also have limited inventory space without paying, which specifically restrict the amount of inventory space which is available in the game. Even if the player obtains items for free within the game, they would have no way of holding onto them without payment.

"Elder scrolls online, and now Fallout 76, creating quality of living issues (inventory space) that are remedied by pay services and subscriptions." (P174)

\section{Game unplayable without spending money}

Sometimes the monetisation of basic quality of life has a harsher manifestation, with the game being unplayable after a certain point without spending money, meaning that although an initial part of the game might be free, the player is literally unable to play the game further than a specific point without a transaction. Players appear to feel particularly misled in situations where they were not informed of this prior to beginning the game.

"Creating an event which has 20 stages. 18 stages of which you can fulfill for free (just spending loads of your time) and for the last 2 you have to pay ingame currency to get the final reward. This is very very 
sneaky tactics. Even if you're notified at the start of the event you still feel like you're being robbed in plain sight." (P705)

\section{Predatory advertising}

Players reported advertising or product descriptions that present incorrect, incomplete, or skewed pictures of what a purchased product entails. This was a common domain within our sample $(N=171)$.

\section{Unrealistic presentation of product}

Players report situations where a product is made out to be something which is not. Sometimes they report this occurring via explicit deceit. Other times the unrealistic presentation of products takes the form of the tactical highlighting of certain features.

"Cosmetic microtransactions in Path of Exile are often announced via showcase videos that use slow motion, an unnaturally zoomed-in camera, and otherwise situations that are impossible or not reflective of actual gameplay" (P706)

"World of Tanks frequently sells premium vehicles for real money by exaggerating the strengths and not discussing the weaknesses of said vehicles." (P9)

\section{Lack of information about conditions of product}

Other times, players report instances of a product being promoted without discussing important additional conditions or aspects that accompany the transaction.

"Mobile games do this a lot. "Free Gems/turns/skins" then you click it and it's "when you buy this starter pack!" (P391)

\section{Aggressive advertising}

This is a widely-reported technique $(N=65)$, which refers to frequent or inconvenient pestering of players to make purchases within the game. Interestingly, these reports appear to be particularly commonly seen in mobile games.

"The most egregious microtransactions come from mobile games. For instance, Candy Crush - it has microtransaction prompts that "get you" right after you were so close to completing a level." (P381)

\section{In-game currency}

This domain covers issues with practices that relate to virtual currency which can only be used within the context of the game world and has no value outside of it.

\section{General existence of in-game currency}

Some players generally find the presence of in-game currencies to be problematic. 


\section{In-game currency disguises actual price}

This refers to situations where translating purchases into an in-game currency, rather than simply using real money, is perceived as obscuring the true price of in-game items and making decision-making harder.

"False currencies in-game obfuscate the cost of purchases, making it difficult to make a reasonable decision as to something's value." (P778)

\section{Multiple currency types cause confusion}

An extreme case of this confusion over currency conversion appears to occur when there are multiple currency types, in which players report being disoriented by the presence of multiple in-game currencies, and believe that this multiplicity disguises the true cost of in-game transactions.

"Multiple premium currencies. These (such as in Heroes of the Storms currency for playing, currency for real money) make it very difficult to gauge what is required to obtain said items without knowing how much you are spending / how much the item costs." (P404)

\section{Fixed purchase rates are unfair}

Video games commonly constrain the specific amounts of real money that can be exchanged for in-game currency. Players felt that the design of these exchanges may be tactical, in order to maximise profits: currency exchange amounts and microtransaction costs are perceived as misaligned in order to encourage increased subsequent spending.

"I think League of Legends again, in how they price their in game currency. It is intentional so that when you spend 110 worth it ends up only allowing you to buy 1.75 items. It feels incredibly blatant that being left with nearly enough for another purchase should encourage more spending." (P591)

\section{Pay to win}

Pay to win was mentioned by respondents often $(N=109)$. This refers to transactions the outcome of which gives players an advantage towards being successful in the game, often at the expense of other players.

\section{Advantage over other players}

Being able to pay for advantage over other players is seen as inherently unfair. This specifically refers to situations where players report that it is unfair when one player has the option to pay for products which are distinctly better than free items and thus give an advantage over players who have not carried out any transactions.

"Anything that makes paying opponents stronger than non paying is unfair. In dominations this is with troops and museum." (P369)

\section{Subscription features}

Another category is subscription features, which involves regular payments to receive additional features which provide one with an advantage. 
"In Fire Emblem Heroes they added a subscription service for \$10.00 a month called "Heros Pass" which basically gave people who buy it, 3 free 5 star Heros, a bunch of quality of life improvements that people had been asking for since launch, higher stats on specific units, and more" (P462)

\section{Boosts}

Boosts refer to instances of being able to pay to progress through a game quicker or earn additional benefits when progressing through. Boosts do not always affect a player's advantage relative to other players.

"Boosters in puzzle games, many free to play mobile games have a set of boosters that extend turns remaining or clear large parts of a level. Candy Crush is the one I played." (P228)

\section{Pay to play competitively}

In some games, although players are technically able to play the game without buying anything, in order to stand a chance in competing against other players they feel pressured to spend. The word 'compete' is often specifically used to refer to this experience.

"Lord's mobile, West game. The games are Pay2Win. If you're not paying tons of real world money you don't have a fair chance to compete with those who are." (P570)

\section{General presence of microtransactions}

Some players believe microtransactions in general are unfair, particularly when implemented within a product that has already been paid for. It also covers reports of unfair pricing of microtransactions.

\section{Microtransactions as a business model}

This category refers to the broad idea that the very concept of revenue being generated through continuous, uncapped monetisation once the player is in the game is unfair.

"Team Fortress 2 and Counter-Strike: Global Offensive. Great games ruined by greed, I can't even think how could a virtual, non-existant [sic] item could cost almost like a used car. Ironically or sadly, the same company who made my favorite game is also the one responsable [sic] to have brought in this system. And TF2 is the ground zero of this." (P223)

\section{Payment mechanisms in paid products}

Games which necessitate an upfront payment and include additional in-game transactions are described in especially negative terms.

"The assassin creed games have that and I turned me away straight from the start. XP boost, skins and other random money sucking stuff that don't make sense after you pay a bunch for the game." (P990)

\section{Overpricing}

Another relevant issue is overpricing: some players feel that the pricing of in-game transactions is too high for the nature of their purchased products. 
"Crash team racing nitro fueled micro transactions are insane. Some karts (cosmetic items) cost upwards of 25 dollars and the game only costs 40 bucks. Fortnite is just as bad with 40-60 dollar skins (cosmetic item) for a free game." (P757)

\section{Other}

This section consists of several techniques which did not naturally fall into the other domains.

\section{Teasers}

This refers to receiving an initial part of an in-game item for free, such as through gameplay, but not being able to fully use it without spending money.

"A harvest diary event where you get bonus rewards for daily missions, and a special character. However, in order to get the character, you need to pay. Non-playing players will just end up with a pile of shards for that character that isn't enough to unlock them." (P729)

\section{Limited time offers}

This code refers to instances where players believe that some products are promoted as only being available for a set amount of time to artificially create a sense of fear of missing out and anxiety in players and push them to engage with the transaction.

"They're time gated so you can only buy one weekly, giving the impression that they're a good deal to buy, but generally aren't worth their contents" (P252).

\section{Battle passes}

Battle passes are a form of video game monetisation which involve paying for a time-specific set of content, which provides within itself additional rewards that can be acquired either by playing the game or in some cases, paying even more additional money. In certain cases, players link this to the exploitation of both player time and money.

"Around 110 for the battle pass where you have to play a lot to get any decent rewards, it traps you into playing that specific game to get your money's worth out of it" (P598).

\section{Dark interface design patterns}

This refers to situations where a game is not merely aggressive in pushing purchases, but the user interface itself is designed in such a way as to manipulate users into carrying out transactions against their intentions.

"Makes it too easy to click - like putting the button to buy under a screen that you have to push ok to advance. I have my buy locks on because of this but if someone has them auto approved they might not even realize they made a purchase" (P444).

\section{Subset of players not affected}

It is also worth noting that a substantial proportion of players $(N=134)$ did not believe that they had been affected by problematic monetisation techniques. This group can be broadly split into three reasons: one category believed they had 
not encountered such techniques at all, one group was aware of these techniques and actively avoided games which they knew employed them, and one group had encountered them and was able to identify them, but because of being able to do so had not engaged in the actual transactions.

A subset of respondents also did not believe that microtransactions in their essence could be unfair or misleading, as they felt players had substantial freedom to not engage with these transactions and as such any choice they made was of their own volition.

"I think I've been given choice by the games and it's completely up to myself if I spend the money or not. There are games that require more in-game transactions but it's my own choice if I would be willing to continue playing the game in a way its company wants me to" (P951).

"Equality or Justice? It's a game, you can just uninstall it. You have no rights to play video games and therefore no expectations of "fair" pricing" (P957).

\section{Loot boxes}

It is also interesting that although we directly made it clear that we were interested in transactions outside of loot boxes, a subset of respondents $(N=85)$ still talked about loot boxes and the various mechanisms employed in promoting and manipulating their outcomes. This may highlight the high degree to which gamers perceive loot boxes to be predatory, and reflects the level of attention which loot boxes have received thus far.

\section{Discussion}

We worked with a large sample of players of digital games to generate a categorisation of monetisation techniques in games which are perceived as being unfair, aggressive, or misleading, and thus potentially not in accordance with consumer protection laws. To our knowledge, this is the only existing such categorisation of microtransactions across both PC and mobile games. The result is thirty-five types of techniques that are perceived by players as problematic across eight domains.

These techniques are split into 8 domains: game dynamics designed to drive spending, product not meeting expectations, monetisation of basic quality of life, predatory advertising, pay to win, in-game currency, microtransactions as a business model, and other (for techniques which did not cluster into any of the other groups). Understanding that these are the mechanisms which players, the primary users of games, may perceive as problematic and therefore having an effect on their game experience, is important for designing games both as products and as experiences. These points will now be addressed in more detail.

\section{Application to consumer protection}

Given the framing of our survey which elicited player perspectives was based on the wording of the Unfair Trading Regulations, our work has some potential implications when considering players of digital games as consumers of products: several of the in-game monetisation techniques described by players could be seen as being in tension with these Regulations. A full outline of this is provided in Table 1, according to what types of microtransactions might be considered as unfair, misleading, or aggressive. 
Misleading: misleading actions include misleading advertising, artificial scarcity, false information, and misleading omissions, in the case of which certain information about the product is withheld.

Unrealistic presentation of product (in predatory advertising)

Lack of information about conditions of product (in predatory advertising)

Product does not incorporate everything a player believes it to (in product does not meet expectations)

Sale of useless products or duplicates (in product does not meet expectations)

Early access content where players end up with something different to what they paid for (in product does not meet expectations).
Commercial practices which contain false information are specifically prohibited under the Unfair Trading Regulations

Information which may be important to making a purchase decision is perceived as being obscured.

Players assert that they have been misled into a purchase through false promises about content.

Players assert that they have been misled into a purchase through false promises about content.

Players assert that they have been misled into a purchase through false promises about content.

Unfair: practices which contravene "the requirements of professional diligence" and "materially distorting or likely to materially distort" consumer spending.

In-game currency

The perception of obfuscation and deliberate confusion, leading consumers to spend more than they may have intended.

Aggressive: impairing "the average consumer's freedom of choice or conduct ... through the use of harassment, coercion or undue influence" in such a way that said consumer takes a transactional decision that they would not otherwise take.

Aggressive advertising (in predatory advertising)

Players reported advertisements on the behalf of industry that may be classified as harassment.

Dark interface patterns (in other)

Players perceived patterns which coercively impaired their freedom of choice regarding spending.

Table 1. Summary of types of microtransactions which could be considered as contravening the Unfair Trading Regulations.

\section{Misleading practices}

Several monetisation practices were described by players in ways that may align with how the Unfair Trading Regulations define 'misleading' practices: misleading actions which include misleading advertising, artificial scarcity, and false information, and misleading omissions, in the case of which certain information about the product is withheld. This largely applies to predatory advertising. For example, players report the unrealistic presentation of products in dishonest ways, such as through tactical highlighting of features or provision of false information, and lack of information about conditions of product, in which case some critical information that may affect engagement with a transaction is withheld until after the transaction is complete. 
Further examples of this are product does not incorporate everything a player believes it to, sale of useless products or duplicates, and early access content where players end up with something different to what they paid for. In all of these instances, players engage with a purchase with a created expectation that is different to the actual product they receive.

\section{Unfair and aggressive practices}

The Unfair Trading Regulations additionally define a generic set of prohibited practices as those which contravene "the requirements of professional diligence" and "materially distorting or likely to materially distort" consumer spending; or are aggressive, which the Regulations define as impairing "the average consumer's freedom of choice or conduct ... through the use of harassment, coercion or undue influence" in such a way that said consumer takes a transactional decision that they would not otherwise take.

One good example of this is the domain of in-game currency. Players perceive in-game currency as being specifically implemented to contribute to their confusion when they are deciding whether to make a purchase, such as in the case of multiple currency types cause confusion and in-game currency disguising actual price, which could fall under 'material distortion'. In some instances, players also believe currency bundle sizes to have been intentionally designed to be of inconvenient size, meaning they end up spending more than they would have liked to acquire the correct amount (fixed purchase rates are unfair).

\section{Unfair or aggressive advertising}

In terms of aggressive practices, the most prominent example in our data were reports of aggressive advertising. This code referred to situations in which players clearly reported behaviour on the behalf of industry that may be classified as harassment. Another example is the practice of dark interface design patterns, relating to in-game interfaces might be seen as a form of aggressive commercial practice as they may impair freedom of choice amongst gamers.

\section{Relationship to previous work}

A direct connection can be seen between our findings and those of King et al. (2019), who investigated the way major games companies have patented designs for systems that are based around encouraging repeat purchases. Interestingly, players within our sample perceive very similar mechanics to the ones identified by King et al. operating under the surface of the games that they play. For example, King et al. discovered that games may present players with time-limited offers with limited information about their contents, which is represented in our sample through limited time offers and lack of information about conditions of transaction. King et al.'s findings in particular draw attention to purposeful system manipulation, such as players being directed into unfair matchups. From our results it appears that the players themselves are clearly aware of said techniques, and do not feel positively about their implementation.

The reported monetisation techniques also encompassed monetary design patterns as outlined by Zagal et al. (2013), who discuss paying to skip and gating access to content. However, Zagal et al. also describe the monetisation of rivalries as an

important dark design pattern, which was not at all present in our sample. Indeed, a surprisingly low number of people commented on social dynamics in relation to monetisation in our work: while things like unfair matchups were discussed, they were referred to from a game-balancing rather than social perspective.

Overlap can also be seen between our categories and the transaction types taxonomy of Windleharth and Lee (2020). In particular, their work recognises premium currency and its potential for misuse, limited time offers, and a variety of techniques which they categorise as 'resources', namely: direct gameplay advantage (in our work, pay to win), powerups (XP boosts), limited content (parts of game locked behind paywalls), and inventory capacity (limited inventory space 
without paying). However, there are many techniques present in our categorisation which are not found in the one of Windleharth and Lee (2000), and vice versa. As such, the two might be used complementarily.

Although several monetisation techniques were prominent across our sample, a considerable number of players commented that they did not believe they had been misled because of their own alertness, or indeed, they had gone one step further and were avoiding the types of games which used such techniques. This may be seen as reassuring, as it suggests that players may be able to identify and reject or resist spending money in problematic ways in games. Yet this was still only a fraction of our sample, which may suggest that far from all players are able to assess and remain objective about monetisation in this way.

\section{Implications}

As discussed above, the Regulations cover a large amount of issues raised by players in terms of aspects of games which they believe have been implemented to drive revenue generation in potentially problematic ways. Indeed, the only domains which have no conceivable overlap with consumer protection are those of microtransactions as a business model, which focuses more broadly on player distaste for the addition of payment mechanisms into a game, and pay to win, which give players in-game advantages for money, and there are some domains which could be interpreted subjectively, such as game dynamics designed to drive spending. Nonetheless, these domains were still identified by players as potentially problematic, and it is important to address this at this point.

Given that in essence, all games are products which are created to make developers revenue, what is the point at which these mechanisms become problematic, rather than purely cumbersome? It is true that all products to an extent use monetisation tactics for which there will be an unhappy proportion of consumers, and indeed, it is the case that any domain confers an advantage by payment. Developer Richard Garfield discusses that 'any hobby you have, you have to invest something', and defends microtransactions as being 'pay to participate' rather than 'pay to win' (Motley, 2018). This is a sentiment echoed by many.

Furthermore, some authors have asserted that the majority of revenue from microtransaction-based models actually comes from a small proportion of spenders, most of whom spend consciously and of their own free will (Paul, 2020). Under this view of monetisation, one might argue that employing the described monetisation techniques meets the developer requirements and is actually not problematic. However, it is unclear (a) what proportion of in-game revenue is actually driven by heavily-involved individuals; and (b) whether this spending is necessarily unproblematic in terms of an individual's overall financial health. Indeed, convenience samples of loot box spenders have found both that relatively few individuals spend heavily on games; and that this spending appears uncorrelated with an individual's reported household earnings(Close et al., 2021). Moreover, there is less knowledge on this regarding other types of microtransactions.

Also, games are arguably a special type of product, due to their immersive properties and potential for longer-term engagement with users making them powerful spaces for possible manipulation, and it is important to understand these spaces and the mechanisms they employ for the minority of players to whom they may pose a risk. Although it is outside the scope of this paper to fully answer where the line between essential for revenue generation and problematic for players truly is, we can provide some thoughts based on our work as to what characteristics of microtransactions could be perceived as problematic, and the reasons behind this.

Firstly, problematic microtransactions have a tangible aspect: they are specific design elements which can be identified within the game. For example, one can clearly see in the case of DLCs which content must be paid for, or with wait timers, how long one must wait if one does not pay. Immediately, this separates away the more nebulous perspectives, such as 
those regarding more generally discussions of fairness, like microtransactions as a business model. Some subjectivity of course remains here - with DLCs, some players may argue that is the core part of the game being locked behind a paywall, whereas others will see it as additional content. This is also where confidence in whether a microtransaction is problematic would grow with the sample size: even in the case of subjective player perspectives, the opinion of 50 players who see the same part of a game negatively carries more weight than that of one person.

Secondly, truly problematic microtransactions will have consequences, and by virtue of that statement, it will be possible to measure these consequences. This therefore directly ties into the previous point about tangibility. If a microtransaction is perceived by players as being problematic but then has no effect on their person, that is of an entirely different category to a microtransaction which is perceived as being problematic and then affects one's wellbeing, financial circumstances, etc., outside of the game also. (There is also a third option, in which a microtransaction does have consequences but is not perceived by players as being problematic; this is addressed in more depth in the 'Limitations' section). Currently, we have very limited understanding regarding the consequences of microtransactions and whether any of them could actually be considered harmful, given a game containing player-perceived problematic mechanics does not necessarily link to reasons behind high player spending. Understanding these consequences could therefore help contextualise microtransactions further.

That is not to say that something which is perceived by players as being problematic and yet only affects the player within the game is necessarily not actually problematic and merely annoying. Such microtransactions could be problematic along a different axis. As mentioned briefly above, players tend to engage with a game for the gameplay experience. It is therefore a player's expectation that the game creates a 'magic circle' which is shielded from economic concerns; one's experience in the game is not dependent on the spending of money (Deterding, 2016; Lin \& Sun, 2011). Indeed, this was a prominent theme in the findings of Lin and Sun (2011), whose subjects discuss issues of fun, quality, and the gameplay balance in the context of player self-perception. The shift from focus on enjoyment and expression values to revenue generation values are therefore disruptive to this 'magic circle'.

This can be further exemplified by drawing a distinction between optional and forced microtransactions; games which allow for play without the need to spend anything and in which microtransactions simply enhance the experience are traditionally perceived better than those where progress is worse or unattainable without any payment. Besides the above point about the integrity of the gameplay experience, this brings to light ideas about the importance of player agency and choice in choosing whether to engage with an in-game payment. Indeed, an example of a game which was highly positively received was Nintendo's (2013) Rusty's Real Deal Baseball, which not only charged only for additional content after the player had already been playing for a while, but allowed players to interact with the in-game characters to haggle down the price of this content (Paul, 2020). Although the eventual price may have been the one intended all along, and Nintendo may have simply been employing a clever marketing technique, the presentation of the transaction in this way allowed the player to retain their perception of the economic upper hand.

This can be discussed in the context of the current work in the category of pay to win. Pay to win elements are partially seen negatively because they create an unnecessary social discrepancy between those who can afford to pay and those who cannot. However, it can be argued that this negative attitude stems from player perceptions of fairness which do not take into account the broader opportunities of equality that in-game payments can offer certain demographics because of their flexibility (Alha, 2020). Yet they are also believed by players to remove the core element of the gameplay experience: there is no challenge or fun in actually completing the game if it can be completed purely by paying, and those aspects are integral to player expectations of a game. Thus, pay to win elements can still be considered as problematic. 
It can be seen that although the microtransaction model should not be immediately denounced, and developers should be able to think about how to maximise revenue and engagement from their products, there is clearly a line to carefully tread when considering how to implement these decisions in a way which does not have consequences for players within or outside of the games. This is therefore a domain which is perhaps too nuanced to be fully covered by consumer protection laws - even though there are some categories which appear to be in misalignment with said laws. To an extent, games can be treated as products like any other, and some domains can be policed directly: for example, in the case of omissions, provision of false information, not including expected content in purchased product, etc. However, there are other issues which cannot be addressed quite as linearly, for example, ideas of fairness and equality (Alha, 2020) which are less prominent in other commercial products. In summary, game monetisation is a complex domain, and may be accordingly best served by a separate set of regulations that needs to be developed through discussion with industry, researchers, players, and ethicists.

The current work also sheds some light on how player perception of game structures might be different to externally-assumed norms. For example, pay-to-win mechanics have been defined as any in-game payment mechanism which gives an advantage (Paul, 2020), and several of the categories our analysis identified would fit into this definition (e.g. aspects of monetisation of basic quality of life). However, players label pay-to-win mechanics as primarily those which provide players with an advantage over others. Understanding such nuances of what players believe to be problematic and why, as well as the language they use to refer to these techniques, is a step forward to a mutually beneficial games industry.

\section{Limitations}

The self-report methodology provides the most appropriate starting point for understanding the player perspective. However, it has one obvious limitation: subjectivity. A player may perceive a mechanism at work whereas in reality the algorithmic underpinnings of a game implement no such mechanism. This may be exacerbated by existing player pre-conceptions of free-to-play games and in-game transactions as 'bad', inferior to alternative payment models (Paul, 2020), leading players to be harsher in their assessment of microtransactions. Nonetheless, this should not strongly influence our results. Even if the monetisation techniques players discussed are influenced by their preconceptions, they are still presented by a large number of players, and as such, deserve attention. Furthermore, they shed light on why players may be biased against in-game payment mechanisms, which ultimately serve the games industry in understanding how to alter these perceptions for the better.

Conversely, using purely the player perspective may also have the opposite effect: players may not be aware of a potentially problematic mechanism that may have adverse consequences. This is another indication of the importance between academic and industry collaboration if we are to reach a maximally ethical, favourable, and profitable solution.

Furthermore, we only investigated the nature of problematic monetisation. A critic may note that the majority of video game monetisation may be entirely unproblematic: fair, unaggressive, and honest - a valid concern. Our interest here was specifically in transactions which had the potential to be negative to the player in some way. However, it may be the case that if we had gathered data investigating a more neutral question, asking about microtransactions in general, we may have uncovered a more holistic picture of how video games make money.

We also did not collect any participant demographics, which means we were not able to control whether the views expressed in the survey were over-representative of any particular group, such as male gamers. Notwithstanding, we believe that the large sample size allows for confidence in taxonomizing specific types of microtransactions that could be problematic across different games, even though this taxonomy should not be seen as exhaustive. 


\section{Future directions}

This work highlights the need for significantly broader research in the area of video game monetisation. Pressing questions of investigation should include demographic analyses which explore who is most engaged with these transactions, and to what extent are these heavily-engaged individuals driving the revenues generated by the video game industry; it should include longitudinal designs that test whether engaging with the forms of monetisation outlined above lead to financial harm to players; and it should include representative sampling strategies to estimate the prevalence of exposure to these techniques. As technology and economics continue to shift and emerge, research should also be aware of upcoming trends, both in games business models - for example, cryptogames (Scholten et al., 2019), which would shift the player-game relationships discussed in this paper. Additionally, it would be of interest to consider specific types of players who have a fundamentally different perspective on game monetisation, such as professional gamers.

It is also vital to note the intersection between this research and contemporary policymaking. Policy debates surrounding loot boxes have currently led governments from around the world to consider how one specific form of monetisation within the video game industry should best be regulated in order to preserve the interests of players. The evidence uncovered here may instead suggest that broader issues should be additionally dealt with: to the best of our knowledge, this study represents the first attempt to map monetisation from the perspective of a large group of players.

Finally, the positive response and level of engagement of participants with this work is a positive indicator that this is important research to the player community. More generally, it points to the fact that involving the stakeholder in problem identification in research can have very positive results, and as such might be useful for future research design in the domain.

\section{Conclusions}

This research suggests that the range of monetisation techniques that players perceive as being problematic are more diverse than has previously been acknowledged. Thirty-five separate forms of monetisation were identified by players as unfair, misleading or aggressive. These results also suggest that several of these monetisation mechanisms may be in tension with existing consumer protection regulations, which points to the need to examine regulation in the context of games. This work also has implications for understanding the player experience in microtransaction-based game models, as well as player perceptions and relationships of games that should be taken into account by developers and regulators alike to create a games industry that is mutually beneficial to all.

\section{Acknowledgements}

We would like to thank Sebastian Deterding for his input at the early stages of design of this study, Leon Xiao for his helpful information on consumer protection regulations, Tom Stafford for his continued support and supervision, and Nicola Zendle for stepping up on short notice to act as the second rater for reliability analysis.

\section{References}

Alha, K. (2020). The Rise of Free-to-Play: How the Revenue Model Changed Games and Playing. Tampere University.

Alha, K., Kinnunen, J., Koskinen, E., \& Paavilainen, J. (2018). Free-to-Play Games: Paying Players' Perspective (pp. 49-58).

Presented at the Proceedings of the 22nd International Academic Mindtrek Conference. 
Alha, K., Koskinen, E., Paavilainen, J., Hamari, J., \& Kinnunen, J. (2014). Free-to-play games: Professionals' perspectives. Proceedings of nordic DiGRA, 2014.

Close, J., Spicer, S. G., Nicklin, L. L., Uther, M., Lloyd, J., \& Lloyd, H. (2021). Secondary analysis of loot box data: Are high-spending "whales" wealthy gamers or problem gamblers? Addictive Behaviors, 117, 106851. Elsevier.

Dahlan, R. (2020). Finding Dark Patterns in Casual Mobile Games Using Heuristic Evaluation.

Deterding, C. S. (2016). Toward Economic Platform Studies (pp. 1-19). Presented at the 12th Annual Game Research Lab Spring Seminar "Money and Games", York.

GOV.UK. (2020). Consumer rights. GOV.UK. Retrieved December 14, 2020, from https://www.gov.uk/consumer-protection-rights

IJzerman, H., Lewis, N. A., Przybylski, A. K., Weinstein, N., DeBruine, L., Ritchie, S. J., Vazire, S., et al. (2020). Use caution when applying behavioural science to policy. Nature Human Behaviour, 4(11), 1092-1094. Nature Publishing Group.

King, D. L., \& Delfabbro, P. H. (2018). Predatory monetization schemes in video games (eg 'loot boxes') and internet gaming disorder. Addiction, 113(11), 1967-1969. Wiley Online Library.

King, D. L., Delfabbro, P. H., Gainsbury, S. M., Dreier, M., Greer, N., \& Billieux, J. (2019). Unfair play? Video games as exploitative monetized services: An examination of game patents from a consumer protection perspective. Computers in Human Behavior, 101, 131-143. Elsevier.

Lin, H., \& Sun, C.-T. (2011). Cash trade in free-to-play online games. Games and Culture, 6(3), 270-287. SAGE Publications Sage CA: Los Angeles, CA.

Lizardi, R. (2012). DLC: Perpetual commodification of the video game. Democratic Communiqué, 25(1).

Mayring, P. (2004). Qualitative content analysis. A companion to qualitative research, 1, 159-176.

McHugh, M. L. (2012). Interrater reliability: The kappa statistic. Biochemia medica: Biochemia medica, 22(3), $276-282$. Medicinska naklada.

Motley, J. (2018, September 5). Artifact, the digital card game from Valve, will require you to spend real money. KnowTechie. Retrieved June 29, 2021, from https://knowtechie.com/artifact-valve-cards/

Narayan, N. (2020, February 4). EA Earns Nearly \$1 Billion from Microtransactions Last Quarter - European Gaming Industry News. Retrieved January 6, 2021, from 
https://europeangaming.eu/portal/latest-news/2020/02/04/63407/ea-earns-nearly-1-billion-from-microtransaction s-last-quarter/

Oh, G., \& Ryu, T. (2007). Game Design on Item-selling Based Payment Model in Korean Online Games. Presented at the DiGRA Conference, Citeseer.

Paul, C. A. (2020). Free-to-play: Mobile Video Games, Bias, and Norms. MIT Press.

Scholten, O. J., Hughes, N. G. J., Deterding, S., Drachen, A., Walker, J. A., \& Zendle, D. (2019). Ethereum crypto-games: Mechanics, prevalence, and gambling similarities (pp. 379-389). Presented at the Proceedings of the Annual Symposium on Computer-Human Interaction in Play.

Schwiddessen, S., \& Karius, P. (2018). Watch your loot boxes!-recent developments and legal assessment in selected key jurisdictions from a gambling law perspective. Interactive Entertainment Law Review, 1(1), 17-43. Edward Elgar Publishing Ltd.

Shokrizade, R. (2013, June 26). Gamasutra: Ramin Shokrizade's Blog-The Top F2P Monetization Tricks. Retrieved December 14, 2020, from https://www.gamasutra.com/blogs/RaminShokrizade/20130626/194933/The_Top_

The Consumer Protection from Unfair Trading Regulations 2008. (2008). . Queen's Printer of Acts of Parliament. Retrieved December 14, 2020, from https://www.legislation.gov.uk/uksi/2008/1277/contents/made

Windleharth, T., \& Lee, J. H. (2020). Taxonomies for Transactions and User Engagement in Mobile Games. Presented at the DiGRA 2020.

Zagal, J. P., Björk, S., \& Lewis, C. (2013). Dark patterns in the design of games.

Zendle, D., Ballou, N., \& Meyer, R. (2019). The changing face of desktop video game monetisation: An exploration of trends in loot boxes, pay to win, and cosmetic microtransactions in the most-played Steam games of 2010-2019. PsyArXiv. Zendle, D., \& Cairns, P. (2018). Video game loot boxes are linked to problem gambling: Results of a large-scale survey. PloS one, 13(11), e0206767. Public Library of Science San Francisco, CA USA.

Zendle, D., Meyer, R., \& Over, H. (2019). Adolescents and loot boxes: Links with problem gambling and motivations for purchase. Royal Society Open Science, 6(6), 190049. The Royal Society. 